Completed questionnaires, which were used to assess the success of the standard and MM-IP processes, were available for 76 patients (standard $n=41$; MM-IP $n=35$ ).

There were no significant differences in patient satisfaction between the two groups and a similarly high percentage of patients in either group were happy with the physician consultation. In addition, there was no significant difference in the anxiety levels experienced by each group. However, more patients in the MM-IP group felt significantly better informed about their disease, treatment, the operative procedure, risks of surgery, postoperative course and chances of success. Those patients who felt most benefit from the MM-IP were educated to an elementary school level. Encouragingly, age and lack of experience using computers were not barriers to the use of the MM-IP.

Despite the unblinded and subjective nature of this study, and the continued need for physician consultation and written informed consent, the authors suggest that the MMP-IP is a valuable addition to the informed consent process.

Original article Bollschweiler E et al. (2008) Improving informed consent of surgical patients using a multimediabased program? Results of a prospective randomized multicenter study of patients before cholecystectomy. Ann Surg 248: 205-211

\section{Increased risk of new onset IBS in individuals with a history of a biliary event}

IBS and biliary disease might be linked; however, it is not clear if IBS is a risk factor for biliary disease or if biliary disease predisposes individuals to the development of IBS. McNally et al. performed a population-based, prospective, cohort study to investigate this potential association. Their results suggest that the diagnosis of a biliary event is associated with an approximate twofold increase in the risk of developing IBS.

Between 1988 and 2003, McNally et al. sent a validated gastrointestinal symptom survey and subsequent follow-up survey to a random sample of Olmsted County residents, MN, USA. The medical history of participants was reviewed to identify individuals with a diagnosis of a biliary event (gallstones or cholecystectomy). A total of 819 eligible individuals responded to both surveys. This group included 726 individuals without IBS and 93 individuals with IBS at initial survey. A previous biliary event was identified in 59 individuals at initial survey. During the 10-year follow-up, no significant association was found between the presence of IBS at initial survey and the subsequent development of biliary events. However, $16 \%$ of individuals with a history of a biliary event at initial survey developed new onset IBS during follow-up compared with $8.5 \%$ of individuals without a biliary event at initial survey or during follow-up (odds ratio 2.2; 95\% Cl $1.1-4.6 ; P=0.03)$.

In light of these findings, McNally et al. suggest that the diagnosis of a biliary event could possibly be used as a disease marker for IBS.

Original article McNally MA et al. (2008) Biliary events and increased risk of new onset irritable bowel syndrome: a population-based cohort study. Aliment Pharmacol Ther 28: 334-343

\section{Role for pH-impedance tests in patients with GERD symptoms but no evidence of acid reflux}

Approximately $50 \%$ of patients with GERDlike symptoms have no evidence of acid reflux on endoscopy and $24 \mathrm{~h} \mathrm{pH}$ testing. New research shows that multichannel intraluminal impedance (MII) $\mathrm{pH}$ testing (which enables the recognition of acid, nonacid and gas reflux) combined with symptom index scoring improves the rate of diagnosis in this difficult-to-treat patient group.

Kline and colleagues investigated the use of $24 \mathrm{~h} \mathrm{MIl} \mathrm{pH}$ testing with symptom index scoring in 37 patients ( 29 women) with symptoms of regurgitation or heartburn who had a negative endoscopy and a normal $24 \mathrm{~h} \mathrm{pH}$ test. $\mathrm{MII} \mathrm{pH}$ testing involved the transnasal insertion of a $\mathrm{pH}$-impedance catheter into the esophagus for $24 \mathrm{~h}$. Patients were asked to continue normal activities and record episodes of heartburn or regurgitation on a data logger. Symptom indices were calculated from patient log data and $\mathrm{MII} \mathrm{pH}$ test results, and associations between symptom and reflux events were determined.

A positive $24 \mathrm{~h} \mathrm{pH}$ test was found in $16 \%$ of patients; $27 \%$ of patients had a positive 